\title{
3 Social Bookmarking
}

Lernziele

In diesem Kapitel lernen Sie, welche Vorteile eine Ablage der Lesezeichen/Bookmarks im Netz haben kann und welche Dienste dafür geeignet sind.

- Delicious

- Diigo

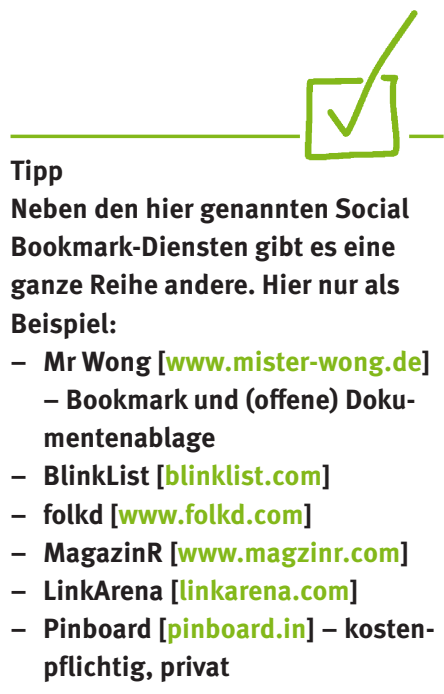

Lesezeichen ablegen ist ein Grundbedürfnis für jene, die sich öfter im Netz bewegen und zumindest ihre Startpunkte und oft besuchten Webseiten ablegen möchten. Meist wird die Sammlung umfangreicher, muss strukturiert und gegebenenfalls mit Hilfe von Schlagwörtern erschlossen werden. Dennoch besteht ein Manko: Man arbeitet heute oft an verschiedenen Geräten, eventuell auch an Geräten mit verschiedenen Browsern, manchmal auch an verschiedenen Orten. Nun, für solche Dinge gibt es Add-ons wie Xmarks [www.xmarks.com], das Lesezeichen und auf Wunsch auch Passwörter zwischen verschiedenen Geräten synchronisiert. So kann man Sammlungen up to date halten, egal wo man arbeitet.

Dennoch ist zu überlegen, ob man darüber hinaus nicht die Lesezeichen in einem Web 2.0-Dienst ablegt, um auf sie von überall her zugreifen zu können. Beispielsweise, wenn man Kurse gibt und auf den Präsentations-Rechner des Kursraumes angewiesen ist. Hier können Sie zwar Xmarks installieren und Ihre Lesezeichen holen mitunter ist dies zeitaufwändig und bremst Sie im Beginn eines Kurses aus! -, wenn Sie sie aber nicht dort lassen wollen, müssen Sie sie mit dem Lesezeichenmanager nach dem Kurs mühselig wieder entfernen. Bei einem Webdienst melden Sie sich ab, schließen die Seite und sind fertig!

Ein weiterer Vorteil solcher Dienste besteht eben in der 2.0-Funktionalität! Sie können hier mit Anderen zusammen arbeiten, sei es, dass Sie die Lesezeichen im selben Account ablegen, sei es, dass Ihre Kolleginnen/Kollegen oder Kursteilnehmer/ innen auf die Sammlung zugreifen können. Oft sind Fragen nach diesem oder jenem Link zu einem Webangebot mit einem kurzen Hinweis beantwortet. Kursteilnehmer/ innen können auch nach dem Kurs auf die Linksammlung zugreifen, wenn sie in einem sozialen Bookmarkdienst abgelegt ist. Ja mehr noch, diese Sammlung wird gegebenenfalls immer weiter von Ihnen aktualisiert!

Darüber hinaus kann man auch mit einigen dieser Webdienste weitere Funktionalitäten in Anspruch nehmen, beispielsweise Webseiteninhalte highlinern und für sich archivieren. Auch mit Endgeräten wie Smartphones oder Tablets können Sie so jederzeit auf Ihre Sammlung zugreifen, entweder über den mobilen Browser oder gegebenenfalls auch eine App.

\subsection{Delicious}

Delicious ist der älteste dieser Dienste, hat zweimal den Eigentümer gewechselt und doch seine Grundfunktionalität beibehalten. Er ist sehr klar aufgebaut, legt die Lesezeichen/Bookmarks chronologisch ab, man erschließt sie mit Hilfe von „tags“, also Schlagwörtern. Außerdem bietet Delicious auf den verschiedensten Ebenen RSSFeeds an, so dass man Aktualisierungen abonnieren und damit zur Kenntnis nehmen kann.

\subsubsection{Neue Inhalte einfügen}

Das Einpflegen von Links in Delicious geht in Sekundenschnelle, wenn man das Bookmarklet installiert hat: Man ist auf einer Webseite, die man bookmarken will, markiert eine Textpassage auf der Seite (1), die den Inhalt der Seite beschreibt, 


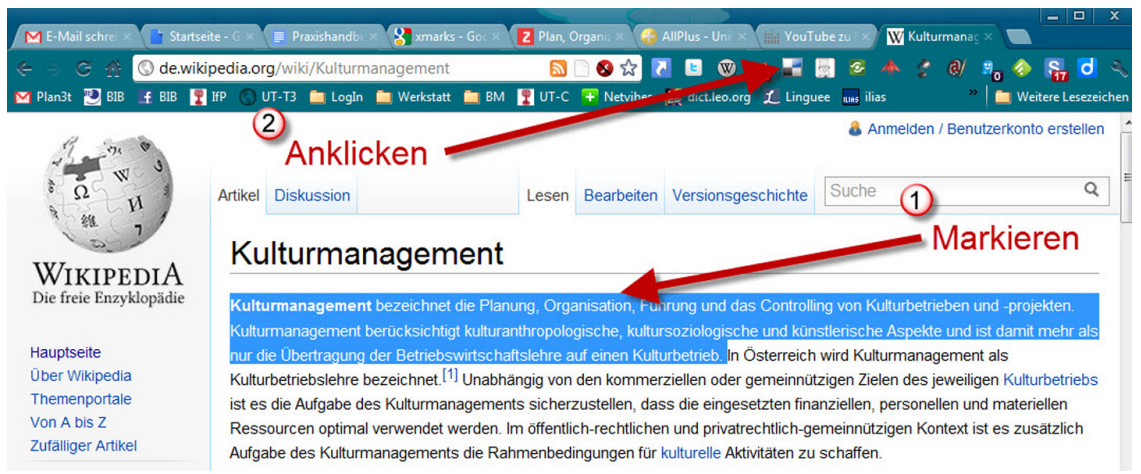

drückt auf das Bookmarklet (2), worauf sich ein Pop-up-Fenster öffnet.

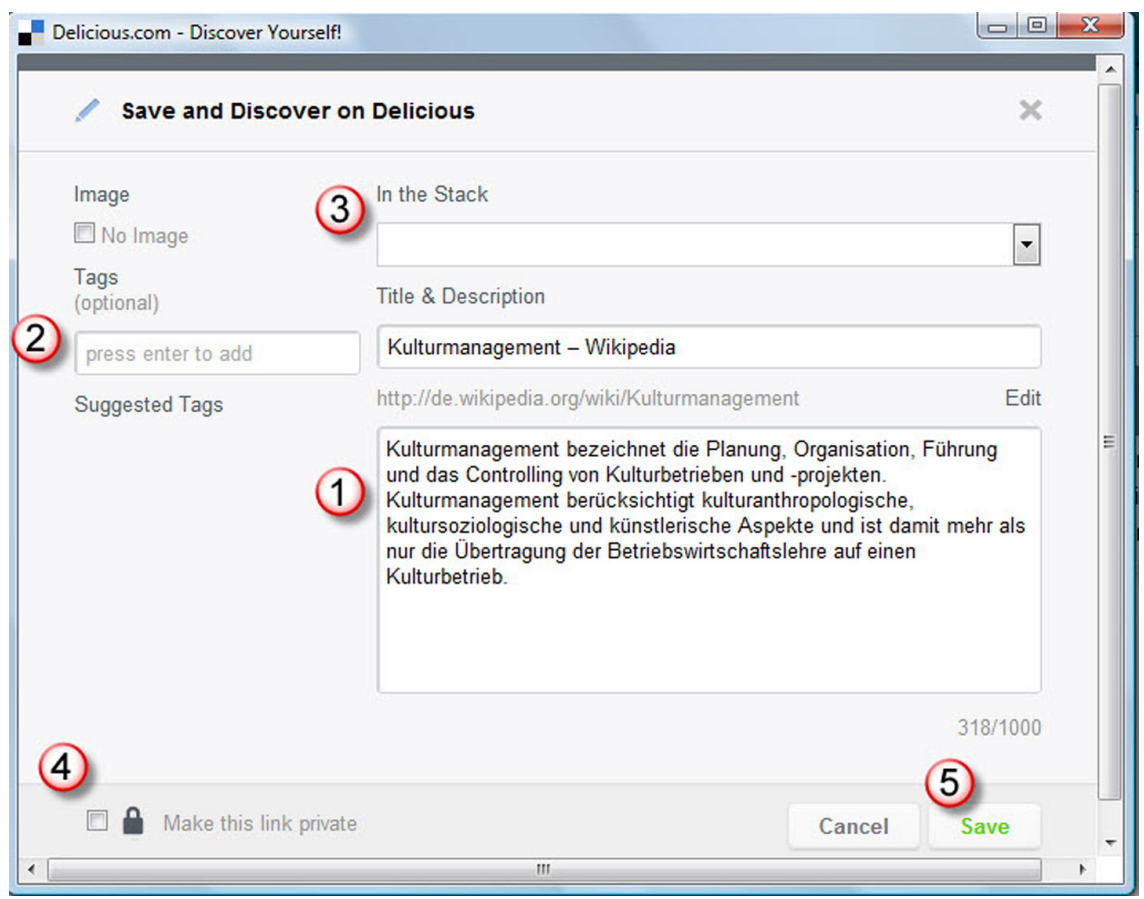

Hier sind bereits Webadresse, Titel und Beschreibung eingefügt. Man kann nun die Beschreibung verändern und ergänzen (1), vergibt einige Schlagwörter (2), kann es bestimmten „Stacks“ (= Themensammlungen, s.u.) zuordnen (3), wählt gegebenenfalls „private“ für eine geschützte Ablage (4), wenn man das Lesezeichen nicht öffentlich ablegen will und speichert das Lesezeichen durch Drücken auf „Save“ (= „Sichern“) ab (5) und fertig!

\subsubsection{Inhalte mit anderen teilen}

Weiter hat man die Option, auf Delicious zusammenzuarbeiten. Wenn Sie und andere je einen Account auf Delicious haben, können Sie sich beispielsweise für die Zusammenarbeit ein Schlagwort überlegen und für Quellen, die Sie für das gemeinsame Projekt ablegen, dieses Schlagwort verwenden. Wenn Sie auf Delicious dieses Schlagwort suchen (in der Form http://www.delicious.com/tag/ $<$ Schlagwort $>$ ), dann sehen Sie alle Einträge, auch wenn sie von verschiedenen Accounts

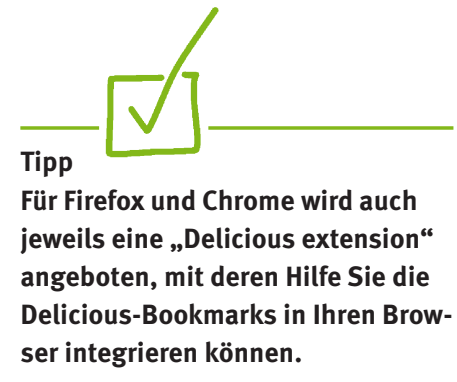

ser integrieren können. 
gemacht wurden. Genauso ist es auch möglich, den RSS-Feed vom Account der Kollegin/des Kollegen zu abonnieren. Oder man bedient zu mehreren denselben Account.

Das Beobachten dessen, was andere auf Delicious ablegen, ist auch über die Registerkarte „Feed“ möglich. Natürlich müssen Sie zunächst andere Accounts abonnieren. Wie geht das? Beispielsweise indem Sie nach bestimmten Themen suchen und Einträge angezeigt bekommen.

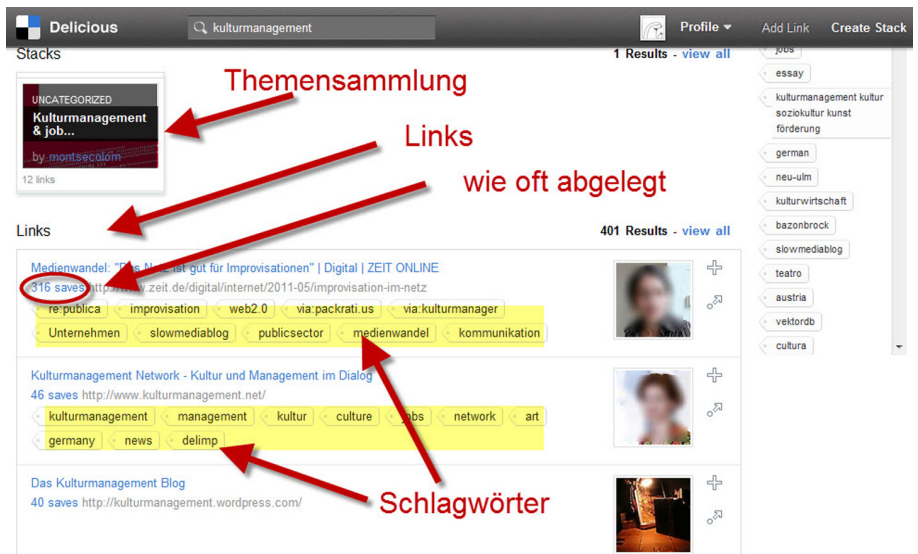

Jetzt können Sie auf die Angabe „x saves“ klicken, die vor der Beschreibung des Eintrags steht,

Delicious

\section{Q Search}

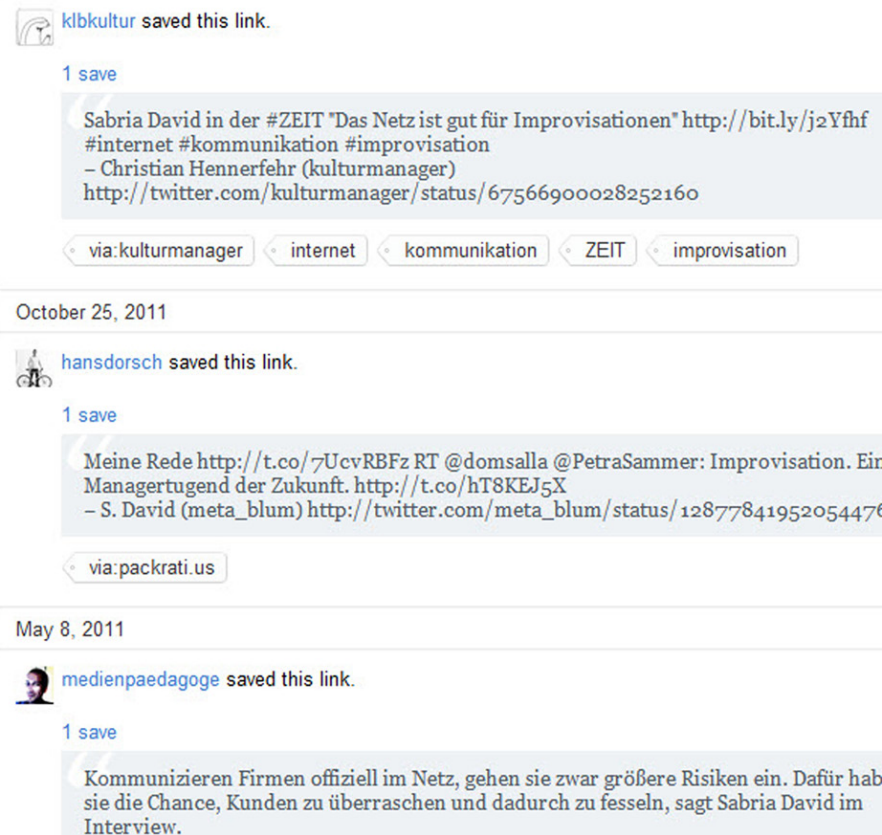

und Sie sehen dann die verschiedenen Delicious-Nutzer, die diesen Link abgelegt haben.

Wenn Sie jetzt wieder auf den in blau gesetzten Benutzernamen klicken, wechseln Sie zu dessen öffentlichen Einträgen. Sagt Ihnen die Sammlung zu, drücken Sie auf den grünen Knopf „Follow“, der sich rechts oben befindet und Sie haben die Ein- 
träge dieses Benutzers abonniert. Diese werden künftig in Ihrem Account unter der Registerkarte „Feed“ dargestellt.

Sie haben mit Delicious oder einem anderen Social Bookmarking-Dienst also nicht nur die Möglichkeit an der Hand, Links abzulegen und von überall her darauf zuzugreifen, sondern auch diese Links in einer thematischen Bündelung für bestimmte Zwecke einzusetzen und mit anderen Personen Links auf Delicious zu teilen.

Die IOS-App Yummy ermöglicht den schnellen Zugriff auf Ihre bei Delicious.com abgelegten Links und ist auch in den mobilen Safari-Browser integrierbar, um Webseiten aus dem Browser in Delicious.com zu speichern. Delicious selbst bietet aber inzwischen auch selbst zum Speichern von Webseiten eine Integration in den mobilen Browser an.

\subsection{Diigo}

Diigo funktioniert ganz ähnlich wie Delicious und unterscheidet sich lediglich durch die eingefügte Kontext-Werbung, die Platz und Aufmerksamkeit kostet. Dennoch ist ein Einsatz von Diigo in Betracht zu ziehen, da es etliche attraktive Zusatzfunktionen bietet. Wenn Sie die Diigo-Toolbar oder „Diigolet“, ein erweitertes Bookmarklet, installiert haben, können Sie

- auf der angezeigten Webseite Textstellen in vier Farben highlighten,

- Notizen hinterlassen,

- sich diese Webseite für spätere Lektüre vormerken (,read it later“-Funktionali-

tät) oder

- die Webseite auf dem Diigo-Server archivieren.

Das bedeutet für Ihren Arbeitsprozess, dass Sie Webseiten schon einmal strukturieren und bearbeiten können (die markierten Stellen werden in Diigo angezeigt, wenn Sie eingeloggt sind!) und so später schneller erfassen, was die Hauptpunkte im Text waren. Ebenso bietet die Archivierungsfunktion die Möglichkeit, flüchtige Texte (z. B. Zeitungsartikel) weiterhin zur Verfügung zu haben und korrekt zitieren zu können, wenn Sie sie irgendwo verarbeiten. Zudem geschieht dies urheberrechtlich korrekt: Nur Sie greifen auf die archivierten Texte zu.

\subsubsection{Erweiterungen}

Diigo bietet eine ganze Anzahl von Werkzeugen, ein einfaches Bookmarklet, ein erweitertes unter dem Namen „Diigolet“ und eine ganze Toolbar. Man kann so, wenn man eingeloggt ist, sehr schnell die verschiedenen Funktionen von Diigo nutzen und Bookmarks ablegen.

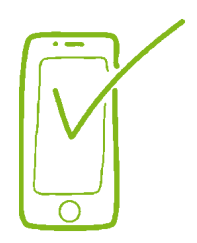

Lese-Tipp

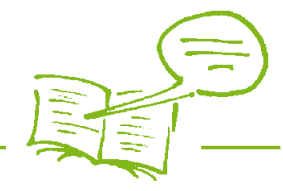

Auf „Professionelle Internetrecherche und Wissensmanagement an Hochschulen“ finden Sie unter [www.recherchieren-im-internet.eu/index.php?id=18] ein Tutorial „Suchresultate festhalten mit Diigo". 


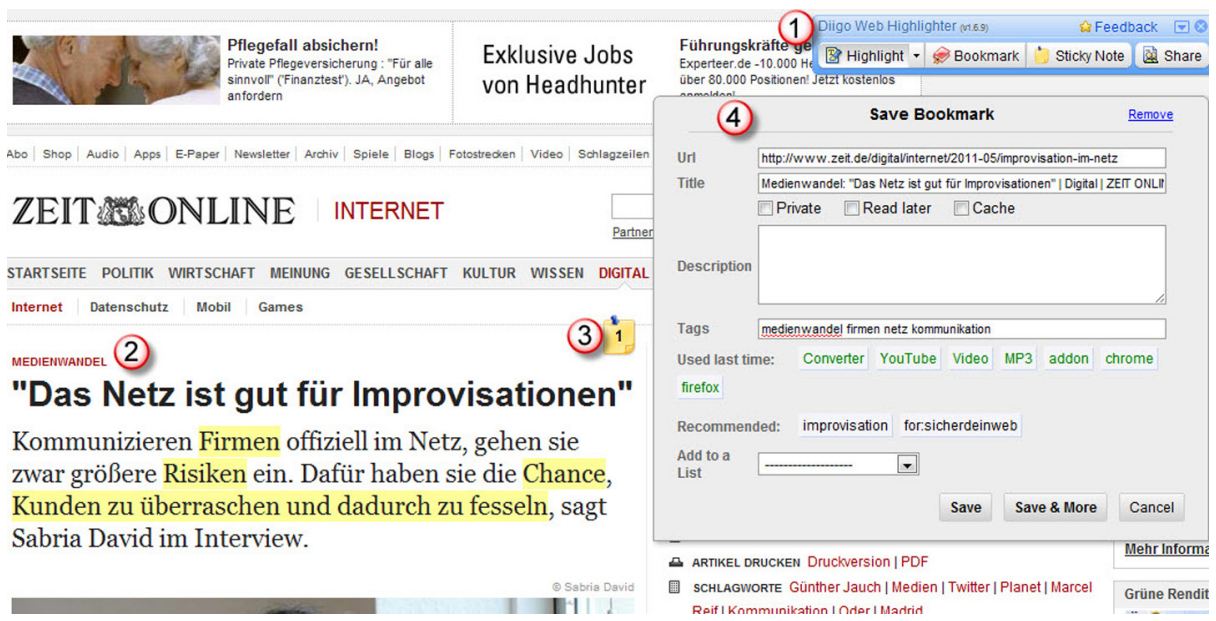

Unter (1) sehen Sie das Auswahlfenster, wenn Sie das Bookmarklet anklicken, bei (2) ist die Quelle zu sehen, in welcher einige Wörter und Passagen hervorgehoben wurden. Unter (3) wurde eine Notiz zu der Seite hinterlegt. Optionen wären gewesen, eine Textpassage zu markieren, was in „Description“ eingefügt worden wäre und wenn das Häkchen bei „Cache“ gesetzt wäre, würde die Webseite mit dem Link gespeichert. Sehen Sie nun, wie der Eintrag dann in der Bookmarksammlung aussieht:

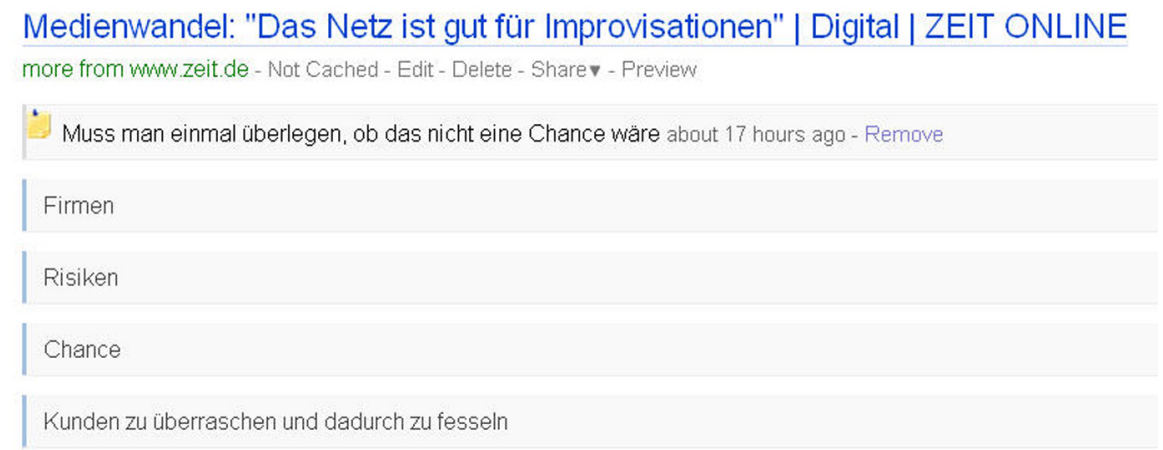

Sehr transparent bezüglich Notiz und Hervorhebungen, nicht wahr? Die Passagen sind auch in Diigo recherchierbar.

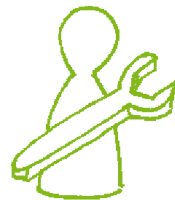

- Suchen Sie eine möglichst einfache Ablage für Links, die auch optisch gut strukturiert ist: Delicious.

- Suchen Sie ein Instrument mit umfangreichen Zusatzfunktionen, wie z. B. zum Markieren von Textstellen und für die Ablage von Dokumenten: Diigo.

- Suchen Sie einen Dienst, der umfangreiche Community-Funktionen bietet wie z. B. Gruppen: Mr Wong. 